\title{
Platelet-rich plasma (PRP) and adipose-derived mesenchymal stem cells: stimulatory effects on proliferation and migration of fibroblasts and keratinocytes in vitro
}

\author{
Talita Stessuk ${ }^{1}$ - Maria Beatriz Puzzi ${ }^{2}$ Elinton Adami Chaim ${ }^{2}$. \\ Paulo César Martins Alves ${ }^{2}$ Erich Vinicius de Paula ${ }^{2}$ - Andresa Forte ${ }^{3}$.

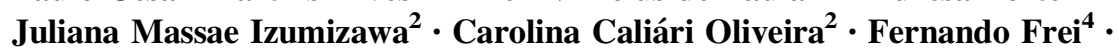 \\ João Tadeu Ribeiro-Paes ${ }^{4,5}$
}

Received: 25 April 2015/Revised: 28 June 2016/Accepted: 1 July 2016/Published online: 9 July 2016

(C) Springer-Verlag Berlin Heidelberg 2016

\begin{abstract}
The clinical use of tissue engineering associated with cell therapy is considered a new alternative therapy for the repair of chronic lesions with potential application in different medical areas, mostly in orthopedic and dermatological diseases. Platelet-rich plasma (PRP) is a rich source of growth factors and cytokines important for wound healing. Adipose-derived mesenchymal stem cells (ADSCs) have shown potential to accelerate the resolution of ulcers, to stimulate cell proliferation, and to benefit the quality of skin repair. This study aims to determine the effect of PRP and conditioned medium (CM) from ADSC on fibroblast and keratinocyte proliferation in vitro. Migration and proliferation assays were performed to evaluate the growth of fibroblasts and keratinocytes in the presence of PRP, CM, and CM + PRP. Significant proliferative stimulation was observed after $48 \mathrm{~h}$ of culture $(p<0.05)$ on mean absorbance of fibroblasts cultured with 10 and $25 \%$ PRP, $100 \% \mathrm{CM}$, and $25 \% \mathrm{PRP}+25 \%$ $\mathrm{CM}$, if compared with control. Keratinocyte proliferation was stimulated after $48 \mathrm{~h}$ in cultures with 25,50 , and $100 \% \mathrm{CM}$, and growth was compared with controls. The migration assay detected a significant migratory stimulus in fibroblasts cultured with $10 \% \mathrm{PRP}+10 \% \mathrm{CM}$ after
\end{abstract}

João Tadeu Ribeiro-Paes

jtrpaes@yahoo.com.br

1 University of São Paulo, USP, São Paulo, Brazil

2 University of Campinas, UNICAMP, Campinas, Brazil

3 CordCell Cell Therapy, São Paulo, Brazil

4 São Paulo State University, UNESP, Campus de Assis, Assis, Brazil

5 Department of Biological Sciences, São Paulo State University, UNESP, Av. Dom Antonio, 2100, Assis, São Paulo 19.806-900, Brazil
$48 \mathrm{~h}$. These in vitro results suggest that PRP and ADSC have therapeutic potential for healing and re-epithelialization of chronic wounds in vivo.

Keywords Fibroblasts $\cdot$ Keratinocytes $\cdot$ Mesenchymal stem cells P Platelet-rich plasma

\section{Introduction}

Chronic skin lesions are a serious worldwide public health problem. In the United States, approximately 6.5 million people suffer of skin lesions, and more than 25 billion dollars are spent each year treating complications related to chronic ulcers [25]. The etiology of leg ulcers consists of diabetes mellitus, dermatological, hematological, rheumatic, and vascular diseases (arterial and venous). It is estimated that $12-25 \%$ of diabetic patients will develop cutaneous foot ulcers throughout their lives [1].

Wound resolution in diabetic patients is impaired by lesion-related physiological and biochemical defects, by abnormalities-in the angiogenic response, in collagen synthesis, in macrophage function, in granulation tissue formation, in growth factor production-and by a decline in fibroblast and keratinocyte proliferation and migration. The environment of the lesion is a chronic inflammatory state characterized by persistent pro-inflammatory cytokines [19]. In this scenario, topical anti-inflammatory strategies associated with growth factors have considerable therapeutic potential.

Wound healing is a complex biological event. Restoring skin integrity requires several cell types, extracellular matrix components, and cytokines [20, 28]. Platelet-rich plasma (PRP), the fraction of the blood plasma with high platelet concentration, has been used to accelerate healing 
in dentistry, orthopedics, dermatology, and plastic surgery [16]. PRP is used for injury regeneration and for tissue repair, because several growth factors essential for healing, such as PDGF, TGF, IGF, and EGF, are concentrated in platelets [23]. In addition, PRP may act as a scaffold for other cell types, such as mesenchymal stem cells (MSCs) [27]. The need for developing therapeutic strategies with potential clinical efficacy, in the context of tissue engineering, has fostered the study of stem cells.

MSCs have immunomodulatory properties and reduce inflammation by secreting cytokines that promote tissue repair in the lesion $[22,29]$. The rationale of this work is based on the existence of a possible beneficial interaction between the PRP and soluble factors from adipose-derived mesenchymal stem cells (ADSCs), on fibroblasts and keratinocytes. This interaction may open a new therapeutic avenue for the treatment of chronic skin ulcers.

\section{Materials and methods}

\section{Biological materials}

PRP was obtained from one unit of whole blood $(450 \mathrm{~mL})$ from a voluntary donation at the Hemocenter from Universidade Estadual de Campinas-UNICAMP (Campinas, São Paulo, Brazil). The volunteer was subjected to serology tests for HIV, HTLV I and II, Chagas, Syphilis, and Hepatitis B and C. Adipose tissue was collected from 30 to 45 (38.5 \pm 6 ) year-old individuals with body mass index (BMI) over than $30 \mathrm{~kg} / \mathrm{m}^{2}$ and who underwent bariatric surgery at the Surgical Center from the Clinical Hospital of UNICAMP. Skin was obtained from patients over 60 undergoing a blepharoplasty procedure at the Ambulatory Surgical Center from the Clinical Hospital of UNICAMP. Prior to each procedure, donors were informed of the study, and invited to participate and to sign the consent form.

\section{Production of platelet-rich plasma}

PRP was obtained as proposed by Jo et al. [11]. Briefly, whole blood was centrifuged at $900 \mathrm{~g}$ for $5 \mathrm{~min}$ to separate the plasma. The supernatant plasma was collected and centrifuged at $1500 \mathrm{~g}$ for $15 \mathrm{~min}$ for platelet concentration [11]. Platelets, leukocytes, neutrophils, and erythrocytes were quantified by the automated hematology analyzer XE500 Sysmex (Sysmex Corporation, Kobe, Japan).

\section{Isolation and culture of ADSC}

ADSCs were isolated following the protocol of Bunnell et al. [2]. Adipose tissue fragments were enzymatically digested with $0.075 \%$ collagenase type I solution (SigmaAldrich, MS, USA) in a $37{ }^{\circ} \mathrm{C}$ shaking water bath for $30 \mathrm{~min}$. Samples were centrifuged at $450 \mathrm{~g}$ for $5 \mathrm{~min}$ to obtain the stromal fraction. The oil and medium containing collagenase were removed. The pellet was resuspended in $1 \mathrm{~mL}$ lysis buffer $\left(0.1 \% \mathrm{KHCO}_{3}, 0.82 \% \mathrm{NH}_{4} \mathrm{Cl}\right.$, and $0.0037 \%$ EDTA.4 Na) and incubated on ice for $10 \mathrm{~min}$. Cells were washed with $20 \mathrm{~mL}$ phosphate buffer Dulbecco's phosphate-buffered saline (DPBS) and centrifuged at $450 \mathrm{~g}$ for $5 \mathrm{~min}$. The cell suspension was filtered through a $70-\mu \mathrm{m}$ cell strainer (Becton-Dickinson, NJ, USA). The filtrate was plated in T25 culture bottles (Sarstedt, Nümbrecht, Germany) at a ratio of $1 \mathrm{~g}$ of adipose tissue for each $6 \mathrm{~cm}^{2}$ [2]. Cells were kept at $37^{\circ} \mathrm{C}$ (in an incubator with $\left.5 \% \mathrm{CO}_{2}\right)$.

\section{Generation of conditioned medium}

Third-passage ADSCs in 80-90 \% confluence, cultured in T75 bottles (Sarstedt, Nümbrecht, Germany), were used to obtain the conditioned medium (CM). The cell surface was washed twice with DPBS phosphate buffer, $\mathrm{pH} 7.4$, supplemented with $1 \%$ penicillin, streptomycin, and L-glutamine (Gibco, New York, USA). $12 \mathrm{~mL}$ of M199 medium supplemented with $1 \%$ penicillin, streptomycin, and L-glutamine was added to each T75 flask. The bottles were incubated at $37{ }^{\circ} \mathrm{C}$ (in an incubator with $5 \% \mathrm{CO}_{2}$ ). After $48 \mathrm{~h}$, the culture medium was aspirated and centrifuged at $300 \mathrm{~g}$ for $10 \mathrm{~min}$ to remove the debris. The supernatant was used as CM [13].

\section{Immunophenotyping of ADSC}

ADSC immunophenotyping was performed by flow cytometry (FACSCalibur-Becton Dickinson, NJ, USA). Third-passage ADSCs were labeled with monoclonal antibodies against CD73 and CD90 (Becton Dickinson, NJ, USA), CD105 (Biolegend, London, UK), the exclusion markers CD34 and CD45 (Becton Dickinson, NJ, USA), HLA-DR and CD19 (Biolegend, London, UK), and CD11b (Exbio, Vestec, Czech Republic). Viability was assessed with the Live and Dead Kit (Invitrogen, NY, USA).

\section{Differentiation of ADSC}

Third-passage ADSCs were cultured with medium provided by StemPro kits (Cibco, NY, USA), following the manufacturer's instructions, and stained with Oil Red O, Alcian Blue, and Alizarin Red (Sigma-Aldrich, MS, USA) to assess adipogenic, chondrogenic, and osteogenic differentiation, respectively. 


\section{Isolation and culture of fibroblasts and keratinocytes}

Skin samples were placed in Petri plates with $0.025 \%$ trypsin $+0.1 \%$ EDTA and fractionated using scissors and clamps. The cell suspension containing dermal and epidermal cells was incubated at $37{ }^{\circ} \mathrm{C}$ and $5 \% \mathrm{CO}_{2}$ for $3 \mathrm{~h}$ and filtered through a $40-\mu \mathrm{m}$ cell strainer (Becton Dickinson, NJ, USA). The filtrate was centrifuged at $400 \mathrm{~g}$ for $10 \mathrm{~min}$. Cells were counted in a Neubauer chamber, plated in T25 culture flasks at a density of $1.0 \times 10^{5}$ cells $/ \mathrm{cm}^{2}$, and incubated at $37{ }^{\circ} \mathrm{C}$ and $5 \% \mathrm{CO}_{2}$. Cells were cultured in M199 medium supplemented with $10 \%$ fetal bovine serum (FBS) (Gibco, NY, USA), $1 \%$ penicillin, streptomycin, and L-glutamine for isolation of fibroblasts. Cells were cultured in keratinocyte-SFM medium (Gibco, NY, USA) supplemented with $10 \%$ FBS and $1 \%$ penicillin, streptomycin, and L-glutamine for isolation of keratinocytes.

\section{ADSC proliferation in the presence of PRP}

ADSCs were seeded ( $n=6$, technical replicates) in 96-well plates (Becton Dickinson, NJ, USA) at a concentration of $2 \times 10^{3}$ cells/well and cultured in M199 medium supplemented with $10 \%$ FBS and $1 \%$ penicillin, streptomycin, and L-glutamine. After $24 \mathrm{~h}$, the medium was removed and replaced with culture medium M199 supplemented with $0 \%$ (negative control), 10, 25, 50, and $100 \%$ PRP (activated with $25 \mu \mathrm{L}$ of $10 \%$ calcium gluconate for each $1000 \mu \mathrm{L}$ of PRP) or $10 \%$ FBS and $1 \%$ penicillin, streptomycin, and L-glutamine. Cell proliferation was assessed after 24 and $48 \mathrm{~h}$ of incubation by adding $10 \mu \mathrm{L}$ of CCK- 8 reagent, following the manufacturer's instructions. Absorbance was measured at $450 \mathrm{~nm}$ for analysis of cell growth.

\section{Skin cells proliferation in the presence of PRP and $\mathrm{CM}$}

Fibroblasts and keratinocytes were seeded $(n=6$, technical replicates) in 96-well plates (Becton Dickinson, NJ, USA) at a concentration of $2 \times 10^{3}$ cells/well. Cells were cultured in M199 medium supplemented with $10 \%$ FBS and $1 \%$ penicillin, streptomycin, and L-glutamine (fibroblasts) and keratinocyte-SFM medium supplemented with $10 \% \mathrm{FBS}$ and $1 \%$ penicillin, streptomycin, and L-glutamine (keratinocytes). After $24 \mathrm{~h}$, the medium was removed and replaced with M199 culture (fibroblasts) or keratinocyte-SFM medium (keratinocytes) supplemented with $0 \%$ (negative control), 10, 25, 50, and $100 \%$ PRP, $10,25,50$, and $100 \% \mathrm{CM}, 10 \% \mathrm{PRP}+10 \% \mathrm{CM}, 25 \%$ $\mathrm{PRP}+25 \% \mathrm{CM}, 50 \% \mathrm{PRP}+50 \% \mathrm{CM}$ or $10 \% \mathrm{FBS}$.
All media was supplemented with $1 \%$ penicillin, streptomycin, and L-glutamine. Cell proliferation was assessed after 24 and $48 \mathrm{~h}$ of incubation by adding $10 \mu \mathrm{L}$ of CCK8 reagent, following the manufacturer's instructions. Absorbance was measured at $450 \mathrm{~nm}$ for analysis of cell growth.

\section{Migration assay}

Cell migration was determined using a scratch assay, as proposed by Liang et al. [14]. Fibroblasts were seeded in 12-well plates at a concentration of $2 \times 10^{5}$ cells/well, in triplicates, and cultured in M199 medium with $0 \%$ (negative control), $10 \%$ PRP, $10 \% \mathrm{CM}, 10 \%$ PRP $+10 \%$ $\mathrm{CM}$ or $10 \%$ FBS. ADSCs were plated in 12-well plates at a concentration of $2 \times 10^{5}$ cells/well, in triplicates, and cultured in M199 medium with $0 \%$ (negative control), $10 \%$ PRP or $10 \%$ FBS. A line was drawn on the confluent cell monolayer using a P200 pipette. Images were acquired on the open space of the plastic. Cell migration was monitored after 24 and $48 \mathrm{~h}$ [14].

\section{Statistical analysis}

The Shapiro-Wilk test was used to analyze the normality of variances. Analysis of variance (ANOVA) followed by Kruska-Wallis and Student Newman-Keuls test for nonparametric data was used to determine significant differences between assays with more than two groups. Statistical significance was set at $p<0.05$. The tests were performed using the BioEstat 5.3 software.

\section{Ethical aspects}

This study was approved by the Ethics Committee for Research of the Faculty of Medical Sciences, UNICAMP, and is registered at Platform Brazil (CAAE 11214513.6. $0000.5404)$.

\section{Results}

\section{Platelet-rich plasma analysis}

The PRP had a platelet concentration factor of $2.050 \pm$ 0.226 and a platelet recovery efficiency of $60.12 \pm 1.77 \%$. A small fraction of leukocytes and neutrophils was obtained after the second centrifugation step (Table 1).

\section{ADSC immunophenotyping}

The immunophenotypic study of third-passage ADSCs showed expression $(\leq 2 \%)$ of exclusion markers, such as 
Table 1 Concentration of platelets and cellular fractions pre- and post-centrifugation
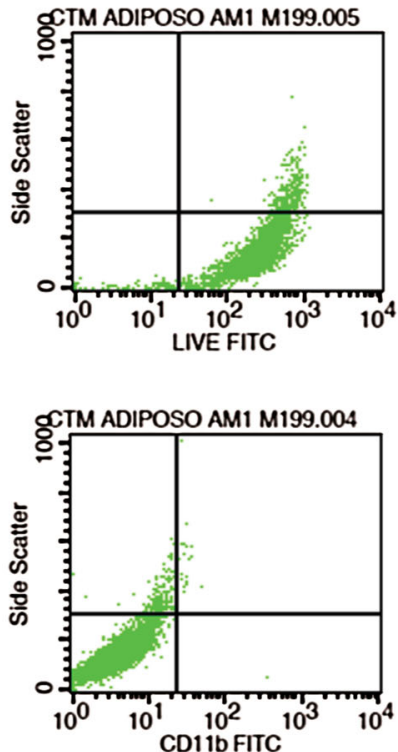

\begin{tabular}{lllll}
\hline Step & $\begin{array}{l}\text { Red blood } \\
\text { cells }\left(10^{6} / \mu \mathrm{L}\right)\end{array}$ & $\begin{array}{l}\text { Leukocytes } \\
\left(10^{3} / \mu \mathrm{L}\right)\end{array}$ & $\begin{array}{l}\text { Neutrophils } \\
\left(10^{3} / \mu \mathrm{L}\right)\end{array}$ & $\begin{array}{l}\text { Platelets } \\
\left(10^{3} / \mu \mathrm{L}\right)\end{array}$ \\
\hline Pre-centrifugation & 3.620 & 3.960 & 1.940 & 232.000 \\
Post-centrifugation & 0.020 & 0.260 & 0.120 & 476.667 \\
\hline
\end{tabular}
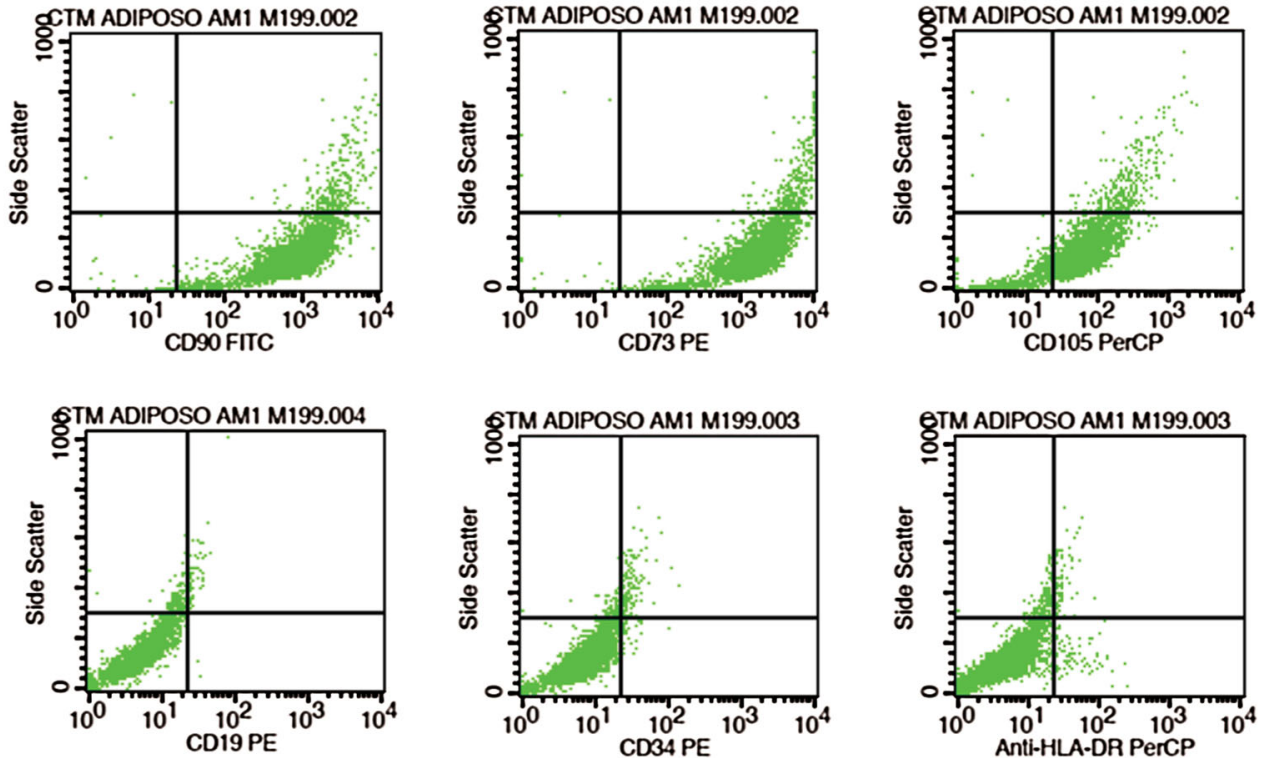

Fig. 1 Immunophenotypic characterization of ADSC by flow cytometry using different surface markers. Positive phenotype ( $\geq 95 \%$ ): CD90, CD73, and CD105. Negative phenotype ( $\leq 2 \%)$ : CD11b, CD19, CD34, and HLA-DR

Fig. 2 Differentiation of ADSC in adipogenic (a Oil red $\mathrm{O}$ staining), chondrogenic (b Alcian blue staining), and osteogenic (c Alizarin red staining) lineages. $10 \times$ magnification (scale bar $50 \mu \mathrm{m})$
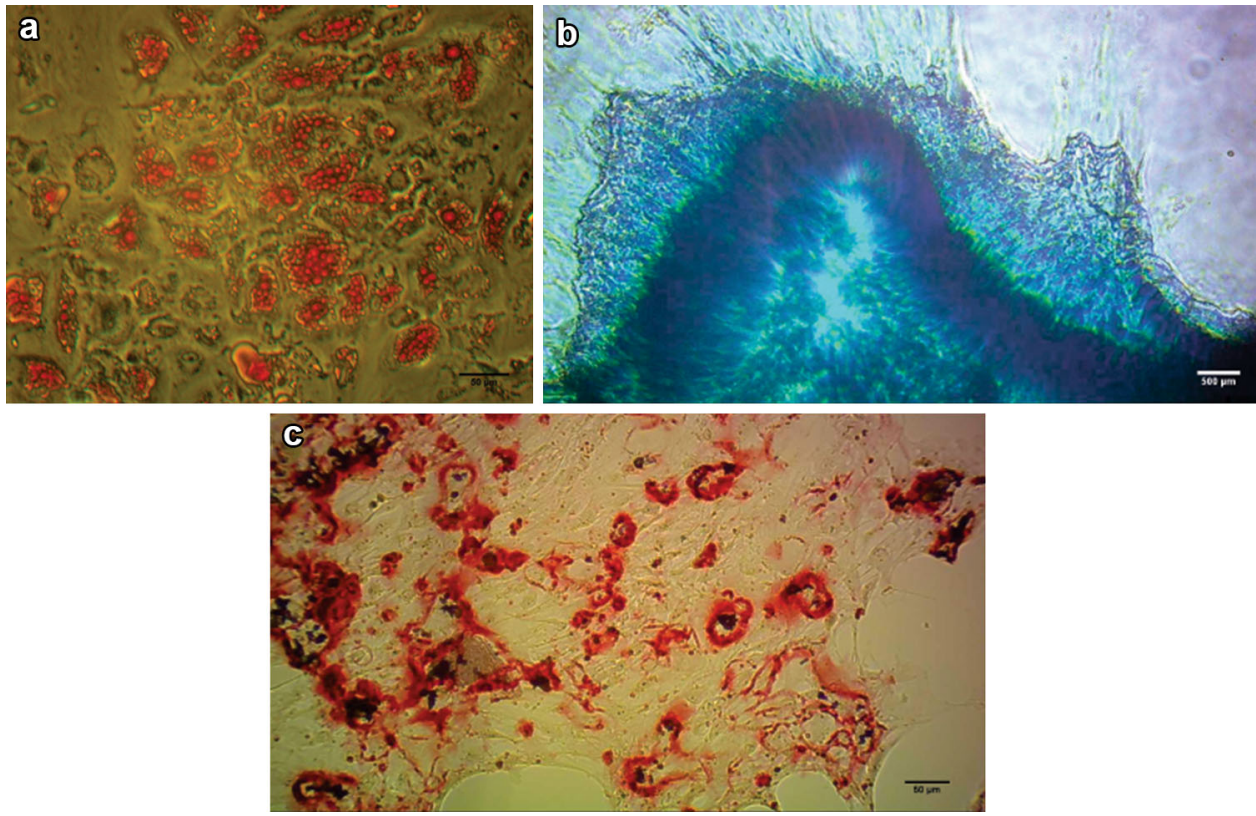

CD34 (0.26\%), CD11b (0.04\%), HLA DR (0.73\%), and CD19 (0.02\%), typical of hematopoietic progenitor cells and leukocytes. In addition, significant positive expression ( $\geq 95 \%$ ) of ADSC characteristic markers was detected, including CD73 (99.78 \%), CD90 (99.80 \%), and CD105 (97.60\%).

Sample viability was $94.14 \%$. Figure 1 shows the flow cytometry results (graphical view). 


\section{ADSC differentiation}

Adipogenic differentiation of ADSCs was observed after 7 days of culture with Oil Red O staining (used for visualization of lipid vacuoles). Chondrogenic differentiation was observed after 14 days of culture with Alcian Blue staining (used to show proteoglycans). Cells differentiated into osteoblasts were stained after 21 days with Alizarin Red (used for labeling calcium deposits), as shown in Fig. 2.

\section{Proliferation of ADSCs under the influence of PRP}

A 48-h proliferation assay was used to determine the influence of PRP on ADSC growth. As shown by the graph in Fig. 3, the concentration of PRP is inversely proportional to ADSC proliferation, i.e., lower PRP concentrations provide higher ADSC growths.

Significant differences could not be detected between ADSC treated with PRP and the negative control after $24 \mathrm{~h}$

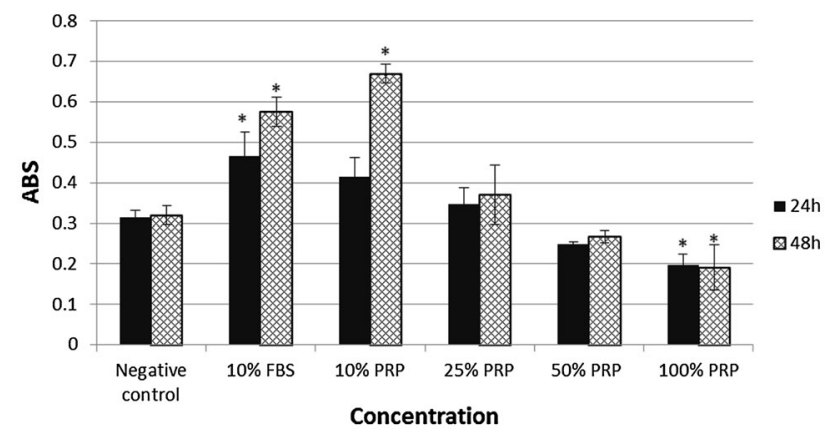

Fig. 3 Growth of ADSC cultured with different PRP concentrations, compared with negative control and FBS. *Statistically significant difference $(p<0.05)$ if compared with negative control for the same respective periods ( 24 or $48 \mathrm{~h}$ )

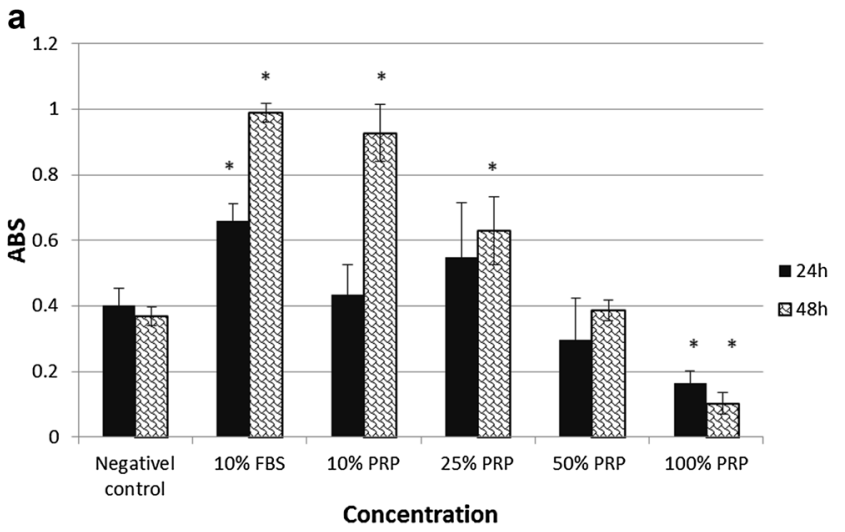

of culture, except for the group cultured with $100 \%$ PRP. This group showed a significant reduction in absorbance reading. Nevertheless, after $48 \mathrm{~h}$, the mean for cells cultured with $10 \%$ PRP was twice as high as in the group without stimulation (negative control), and this difference was statistically significant (Fig. 3).

\section{Proliferation of skin cells under the influence of PRP and $\mathbf{C M}$}

A 48-h proliferation assay (based on absorbance reading) was used to evaluate the influence of PRP and CM from ADSC on fibroblast and keratinocyte growth. As shown by the graphs in Figs. 4 and 5, lower PRP concentrations provide a greater stimulus for fibroblast proliferation and keratinocyte proliferation.

Significant differences could not be detected between cells cultured with PRP and the negative control after $24 \mathrm{~h}$ of culture, except for the group cultured with $100 \%$ PRP. This group showed a significant reduction in absorbance reading for fibroblasts (Fig. 4a) and keratinocytes (Fig. 4b).

Nevertheless, after $48 \mathrm{~h}$, the means for fibroblasts cultured with 10 and $25 \%$ of PRP were 2.51 and 1.7 times higher than the group without stimulation (negative control), and these differences were statistically significant. Fibroblasts cultured with $100 \%$ PRP maintained a difference even after 48 h (Fig. 4a). Significant differences could not be detected between keratinocytes treated with PRP and without stimulation after $48 \mathrm{~h}$ (Fig. 4b).

The graphs in Fig. 5 show that higher CM concentrations provide a greater stimulus for fibroblast proliferation and, particularly, for keratinocyte proliferation.

Statistically significant differences were observed between fibroblasts cultured with $50 \% \mathrm{CM}$, keratinocytes

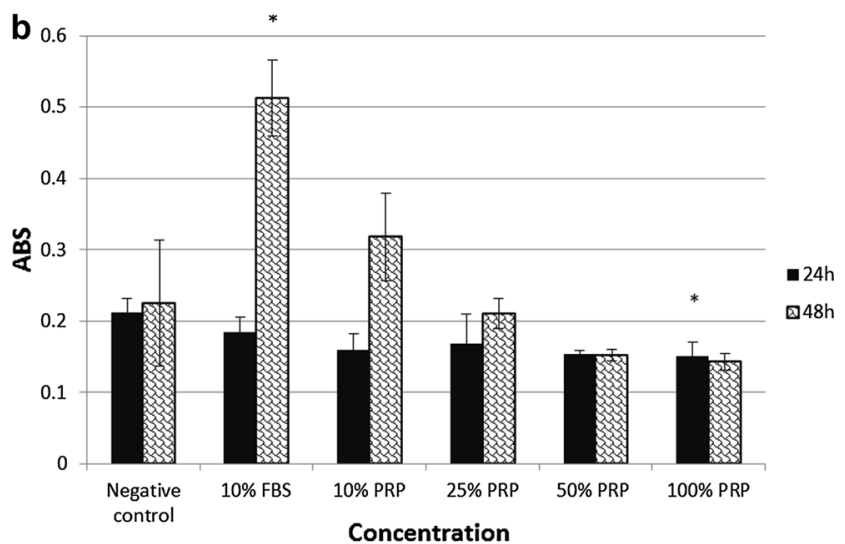

Fig. 4 Growth of a fibroblasts and b keratinocytes cultured with different PRP concentrations, compared with negative control and FBS. *Statistically significant difference $(p<0.05)$ compared with negative control for the same respective periods $(24$ or 48 h) 

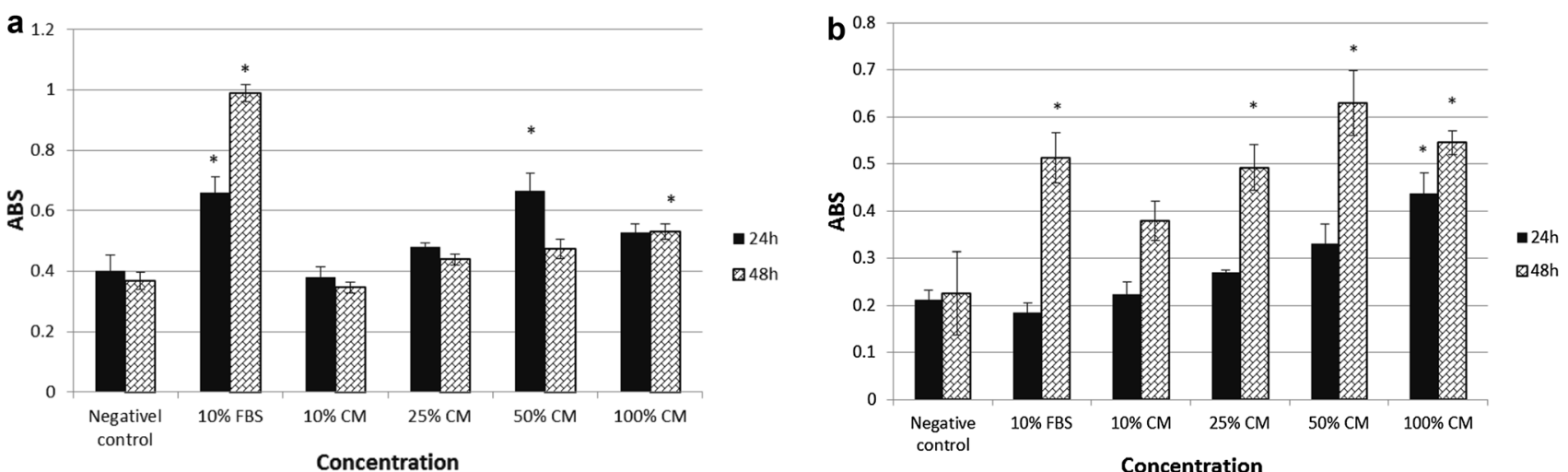

Fig. 5 Growth of a fibroblasts and b keratinocytes cultured with different CM concentrations, compared with negative control and FBS $*$ Statistically significant difference $(p<0.05)$ compared with negative control for the same respective periods $(24$ or $48 \mathrm{~h})$
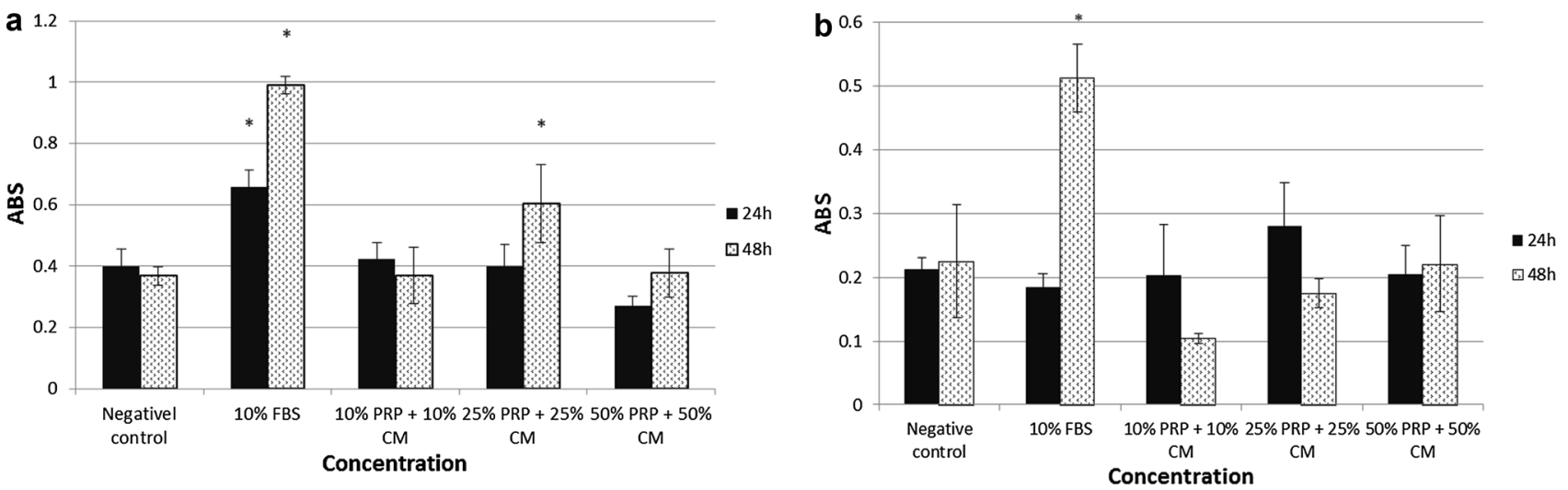

Fig. 6 Growth of a fibroblasts and $\mathbf{b}$ keratinocytes cultured with different PRP and CM concentrations, compared with negative control and FBS. *Statistically significant difference $(p<0.05)$ compared with negative control for the same respective periods $(24$ or $48 \mathrm{~h})$

cultured with 50 and $100 \% \mathrm{CM}$, and the negative control after $24 \mathrm{~h}$ of culture. Fibroblasts cultured with $100 \% \mathrm{CM}$ (Fig. 5a), and keratinocytes cultured with 25, 50, and $100 \%$ CM (Fig. 5b) showed statistically significant differences compared with the negative control after 2 days of culture.

As shown by the graphs in Fig. 6, fibroblast proliferation and keratinocyte proliferation were, to a certain extent, stimulated when only half the culture medium was supplemented with an equal proportion of PRP and CM.

Significant stimulation was observed after $48 \mathrm{~h}$ in fibroblasts cultured with $25 \% \mathrm{PRP}$ and $25 \% \mathrm{CM}$ (Fig. 6a). Higher stimulation of keratinocyte proliferation, although not significant, was observed after $24 \mathrm{~h}$ of cultivation (Fig. 6b).

\section{Migration assay}

A scratch assay was used to determine the influence of PRP and $\mathrm{CM}$, derived from ADSC, on fibroblast migration. The stimulation promoted by PRP on ADSC migratory potential was also examined. Images of lesions made in vitro on the cell monolayer were acquired and the migration quantified after 24 and 48 h, as shown in Figs. 7 and 8.

Quantitative analysis of scratch closure, performed by analyzing the images on ImageJ, showed a statistically significant difference only for ADSC cultured with $10 \%$ PRP, compared with the group without stimulation (negative control) after 24 and $48 \mathrm{~h}$ (Table 2).

The migratory stimulus was significantly different in fibroblasts cultured with $10 \% \mathrm{PRP}+10 \% \mathrm{CM}$ (compared with the negative control) after $48 \mathrm{~h}$ (Table 3).

\section{Discussion}

In this study, we investigated the effect of PRP in ADSC, aiming to identify a repair process with growth factors that is more efficient than conventional treatments, including a powerful antinociceptive activity linked to lipid fraction of PRP [4] for treating chronic cutaneous ulcers $[5,7,21]$. 
Fig. 7 Scratch assay on ADSC cultured without supplement/ negative control $(\mathbf{a}-\mathbf{c}), 10 \%$ FBS (d-f), and $10 \%$ PRP (g-i). Images captured at $0 \mathrm{~h}(\mathbf{a}, \mathbf{d}, \mathbf{g})$, $24 \mathrm{~h}(\mathbf{b}, \mathbf{e}, \mathbf{h})$, and $48 \mathrm{~h}(\mathbf{c}, \mathbf{f}, \mathbf{i})$ following a tracer on the ADSC monolayer
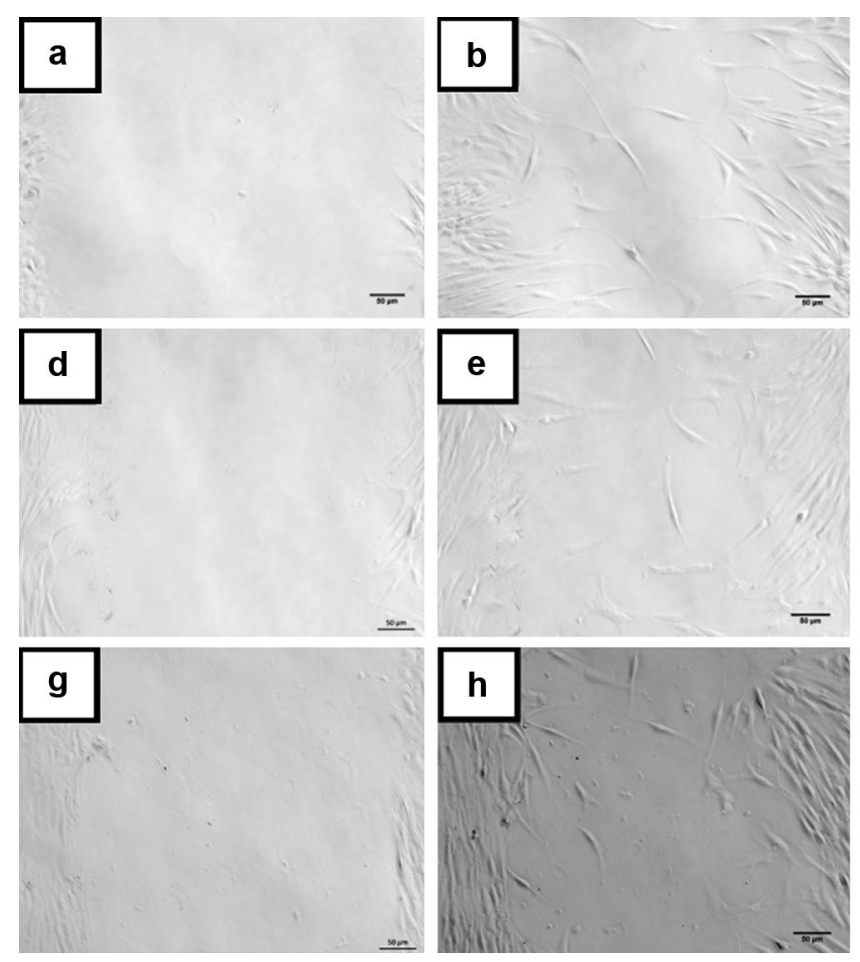
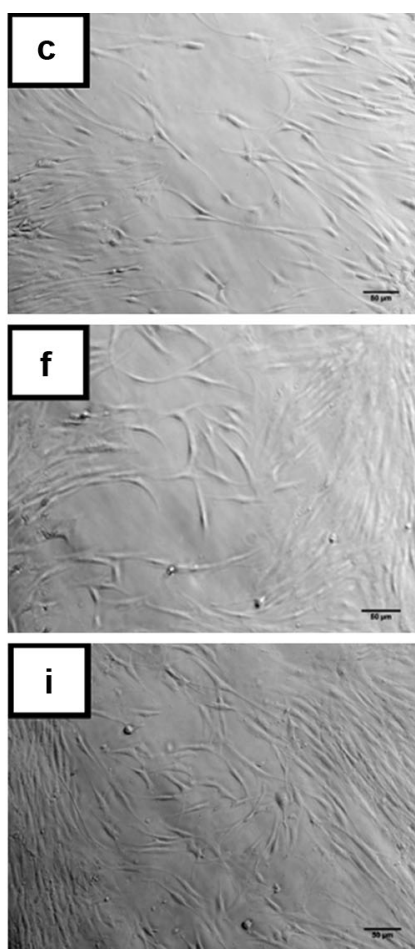

ADSCs were cultured with different PRP concentrations in the absence of FBS. The results showed that there was a proliferative and migratory stimulus in ADSC cultured with $10 \%$ PRP after $48 \mathrm{~h}$, compared with negative control. An inverse dose-dependent relationship was observed, i.e., higher PRP concentrations provided lower proliferative means, as shown in Fig. 3 (proliferation) and Fig. 8 (migration). These results are consistent with previous studies reporting significant proliferative stimulation of cultured MSCs with up to $10 \% \operatorname{PRP}[6,10,12,15]$.

In in vitro cultures, higher PRP concentrations limit cell growth, as platelets release a complex protein composition with a negative regulatory activity that may reduce the effects of growth factors [9]. Furthermore, Mills et al. [17] proposed that when the proteolytic enzymes found in PRP - collagenase, elastase, cathepsin, and acid phosphatase-are highly concentrated, they contribute to cell growth inhibition.

To determine how the skin responds to growth factors, keratinocytes and fibroblasts were cultured with different concentrations of PRP, CM or both. Significant inhibition, similar to ADSC, was observed after $24 \mathrm{~h}$ of incubation with high PRP concentrations, as shown in the graphs of Figs. 4 and 5. Distinct reports in the literature refer to the proliferative stimulus produced by PRP. Kakudo et al. [12] cultivated dermal fibroblasts with different PRP concentrations and saw that after 24 h, 10 and $20 \%$ PRP inhibited proliferation. Proliferative stimulation, however, was only observed on the 7 th day in culture with $10 \%$ PRP. The authors used buffy coat and a large amount of platelets $\left(132.26 \times 10^{4} / \mu \mathrm{L}\right)$, approximately three times higher than the amount used in this study $\left(476.66 \times 10^{3} / \mu \mathrm{L}\right)$. In accordance with data obtained by this study, Kakudo and colleagues found that no PRP proliferative stimulus occurs within $24 \mathrm{~h}$ for fibroblasts. However, in contrast with Kakudo et al. [12], the present study shows that low concentrations of PRP produce no inhibitory effect, even with minimal leukocyte and neutrophil fractions.

In a recent study, Shan et al. [21] reported that the best stimulus for cell growth is achieved with $2 \times 10^{6}$ platelets/ $\mu \mathrm{L}$. However, the authors performed leukodepletion of the PRP and achieved significant growth in human keratinocytes (HaCaT) within $24 \mathrm{~h}$ of cultivation with $10 \%$ PRP. These results indicate that higher PRP platelet concentration and absence of leukocytes may contribute to the proliferation of fibroblasts and keratinocytes in a short period of time. Fibroblasts appear to respond better to PRP, while keratinocytes have shown a negative apparent specific growth rate when cultured in low concentrations of PRP, in a dependent manner [26]. Therefore, the absence of proliferative stimuli in keratinocytes exposed to PRP observed in the present study may be related to the presence of leukocytes and neutrophils, even if in minimum fractions, and to the smaller amount of platelets in the PRP.

$\mathrm{CM}$ provided a proliferative stimulus for fibroblasts and keratinocytes within $24 \mathrm{~h}$. The higher the CM concentrations, the greater were the proliferative stimulus. The CM derived from ADSC contains a fraction that is rich in 
Fig. 8 Scratch assay on fibroblasts cultured without supplement/negative control $(\mathbf{a}-\mathbf{c}), 10 \%$ FBS $(\mathbf{d}-\mathbf{f}), 10 \%$ CM $(\mathbf{g}-\mathbf{i}), 10 \%$ PRP $(\mathbf{j}-\mathbf{l})$, and $10 \% \mathrm{CM}+10 \% \operatorname{PRP}(\mathbf{m}-\mathbf{o})$. Images captured immediately at $0 \mathrm{~h}(\mathbf{a}, \mathbf{d}, \mathbf{g}, \mathbf{j}, \mathbf{m}), 24 \mathrm{~h}(\mathbf{b}, \mathbf{e}, \mathbf{h}$ $\mathbf{k}, \mathbf{n})$, and $48 \mathrm{~h}(\mathbf{c}, \mathbf{f}, \mathbf{i}, \mathbf{l}, \mathbf{o})$, following a tracer on the fibroblasts's monolayer
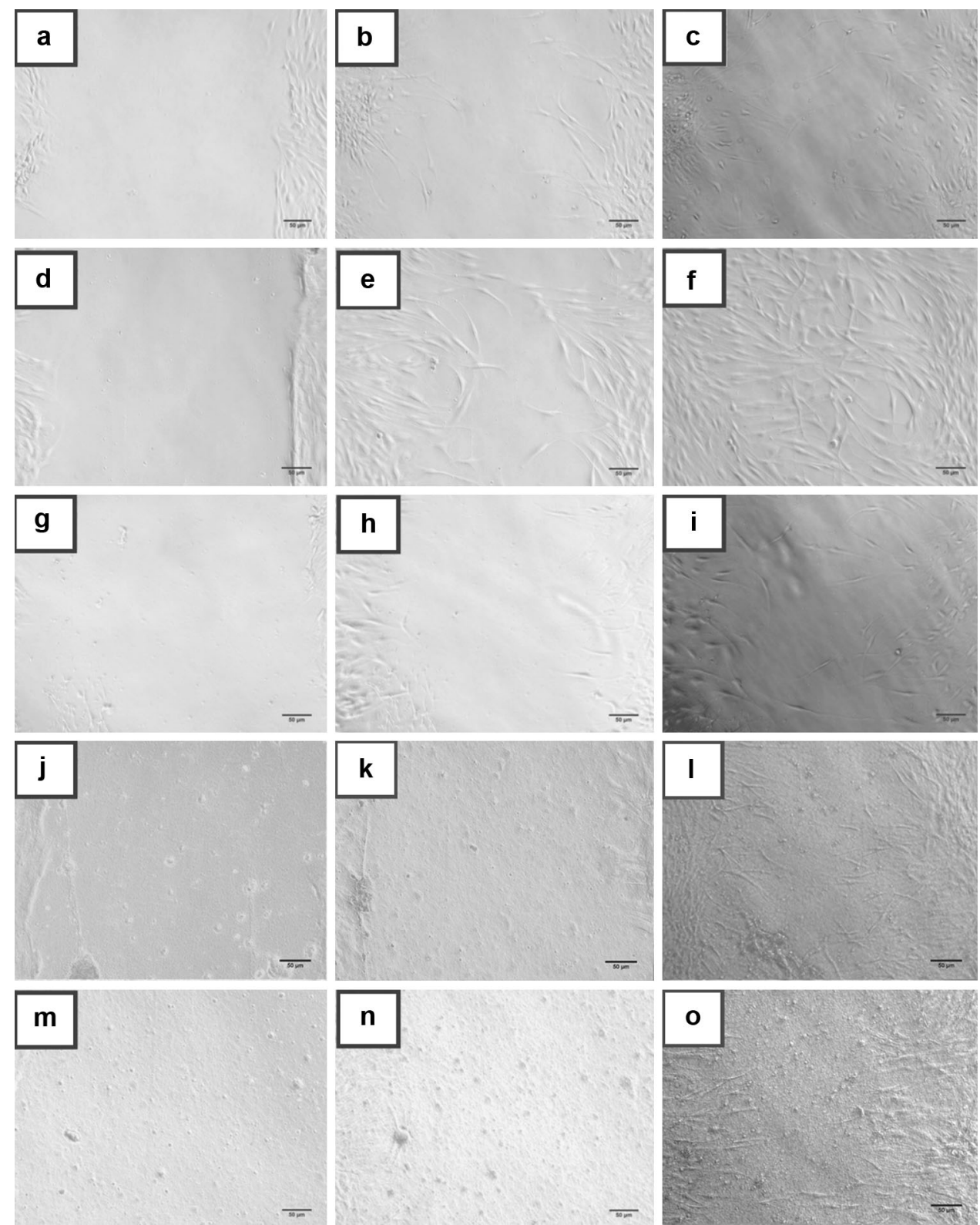

Table 2 Surface area (means percentage) occupied by ADSC after 24 and $48 \mathrm{~h}$

\begin{tabular}{lll}
\hline Group & $\begin{array}{l}24 \mathrm{~h} \\
\text { Mean } \pm \mathrm{SD}\end{array}$ & $\begin{array}{l}48 \mathrm{~h} \\
\text { Mean } \pm \mathrm{SD}\end{array}$ \\
\hline Control & $10.48 \pm 3.63^{\mathrm{a} *}$ & $11.89 \pm 2.68^{\mathrm{a} *}$ \\
$10 \%$ FBS & $16.65 \pm 3.48^{\mathrm{b}}$ & $18.56 \pm 4.46^{\mathrm{b}}$ \\
$10 \%$ PRP & $17.07 \pm 6.47^{\mathrm{b}}$ & $20.02 \pm 2.30^{\mathrm{b}}$
\end{tabular}

* Letters indicate results of pairwise comparisons among means. Means with the same letter (within columns) do not differ significantly $(p>0.05)$
Table 3 Surface area (means percentage) occupied by fibroblasts after 24 and $48 \mathrm{~h}$

\begin{tabular}{lcc}
\hline Group & $\begin{array}{l}24 \mathrm{~h} \\
\text { Mean } \pm \mathrm{SD}\end{array}$ & $\begin{array}{l}48 \mathrm{~h} \\
\text { Mean } \pm \mathrm{SD}\end{array}$ \\
\hline Control & $5.67 \pm 1.76^{\mathrm{a} *}$ & $8.25 \pm 3.04^{\mathrm{a} *}$ \\
$10 \% \mathrm{FBS}$ & $13.69 \pm 3.65^{\mathrm{b}}$ & $22.02 \pm 4.11^{\mathrm{b}}$ \\
$10 \% \mathrm{CM}$ & $7.80 \pm 2.98^{\mathrm{a}}$ & $11.05 \pm 5.83^{\mathrm{a}}$ \\
$10 \%$ PRP & $5.31 \pm 2.05^{\mathrm{a}}$ & $13.02 \pm 3.22^{\mathrm{a}}$ \\
$10 \%$ PRP $+10 \% \mathrm{CM}$ & $5.83 \pm 1.74^{\mathrm{a}}$ & $17.78 \pm 2.64^{\mathrm{b}}$ \\
\hline
\end{tabular}

* Letters indicate results of pairwise comparisons among means. Means with the same letter (within columns) do not differ significantly $(p>0.05)$ 
soluble factors with paracrine action on fibroblasts and keratinocytes, such as EGF, FGF, KGF, IGF-1, VEGF, and PDGF [3], important cytokines for repair of skin ulcers. Zhao et al. [30] showed that fibroblast proliferation depends on EGF and bFGF, which are present in CM and in PRP. This could explain the cell expansion observed in this study with $25 \%$ PRP $+25 \%$ CM (Figs.6, 8), which proposes a possible synergistic action.

Park et al. [18] found that $10 \%$ PRP stimulates fibroblast migration within $24 \mathrm{~h}$. However, similar to the proliferation assays, the authors used a PRP with a platelet concentration that is three-fold the one used in this study. The low CM concentration was also not sufficient to stimulate migration. Nevertheless, higher CM concentrations, such as $50 \% \mathrm{CM}[8]$ and $100 \% \mathrm{CM}$ [24], induce proliferation/migration. Our results showed that fibroblasts do not exhibit a significant migration behavior when cultured with $10 \%$ PRP. On the other hand, fibroblast migration seems to rely on bFGF, VEGF, and PDGF [30]. This could explain why the migratory stimulus is observed only up on combination of PRP and CM, at low concentrations, indicating a synergistic or additive action.

In summary, the results of this study show that low PRP concentrations stimulate proliferation and migration of ADSC and fibroblasts in vitro. Furthermore, keratinocyte proliferation can be stimulated by ADSC paracrine action. These results propose an association between ADSC and PRP soluble mediators, which could potentially be used to promote healing and re-epithelialization in cutaneous ulcers.

Acknowledgments The authors thank the Fundo de Apoio à Dermatologia do Estado de São Paulo-FUNADERSP-Sebastião Sampaio. TS was financially supported by the Coordenação de Aperfeiçoamento de Pessoal de Nível Superior (CAPES-Brazil). JMI was financially supported by the Conselho Nacional de Desenvolvimento Científico e Tecnológico (CNPq-Brazil).

\section{Compliance with ethical standards}

Conflict of interest The authors declare that they have no conflict of interest.

Ethical approval All procedures performed in studies involving human participants were in accordance with the ethical standards of the institutional and/or national research committee and with the 1964 Helsinki declaration and its later amendments or comparable ethical standards.

Informed consent Informed consent was obtained from all individual participants included in the study.

\section{References}

1. Brem H, Sheehan P, Rosenberg HJ et al (2006) Evidence-based protocol for diabetic foot ulcers. Plast Reconstr Surg 117(7 Suppl):193S-209S
2. Bunnell BA, Flaat M, Gagliard C et al (2008) Adipose-derived stem cells: isolation, expansion and differentiation. Methods 45:115-120

3. Chen L, Tredget EE, Wu PY et al (2008) Paracrine factors of mesenchymal stem cells recruit macrophages and endothelial lineage cells and enhance wound healing. PLoS One 3:e1886. doi:10.1371/journal.pone.0001886

4. Descalzi F, Ulivi V, Cancedda R et al (2013) Platelet-rich plasma exerts antinociceptive activity by peripheral endocannabinoidrelatd mechanism. Tissue Eng Part A 19:2120-2129

5. Driver VR, Hanft J, Fylling CP et al (2006) A Prospective, randomized, controlled trial of autologous platelet-rich plasma gel for the treatment of diabetic foot ulcers. Ostomy Wound Manag 52:68-87

6. Formigli L, Benvenuti S, Mercatelli R et al (2012) Dermal matrix scaffold engineered with adult mesenchy-

mal stem cells and platelet rich plasma as a potential tool for tissue repair and regeneration. J Tissue Eng Regen Med $6: 125-134$

7. Gentile P, Orlandi A, Scioli MG et al (2012) Concise review: adipose-derived stromal vascular fraction cells and platelet-rich plasma: basic and clinical implications for tissue engineering therapies in regenerative surgery. Stem Cells Transl Med $1: 230-236$

8. Hu L, Zhao J, Liu J et al (2013) Effects of adipose stem cell conditioned medium on the migration of vascular endothelial cells, fibroblasts and keratinocytes. Exp Ther Med 5:701-706

9. Hsu CW, Yuan K, Tseng CC (2009) The negative effect of platelet-rich plasma on the growth of human cells is associated with secreted thrombospondin-1. Oral Surg Oral Med Oral Pathol Oral Radiol Endod 107:185-192

10. Huang Q, Wang YD, Wu T et al (2009) Preliminary separation of the growth factors in platelet-rich plasma: effects on the proliferation of human marrow-derived mesenchymal stem cells. Clin Med J (Engl) 122:83-87

11. Jo CH, Roh YH, Kim JE et al (2013) Optimizing platelet-rich plasma gel formation by varying time and gravitational forces during centrifugation. J Oral Implantol 39:525-532

12. Kakudo N, Minakata T, Mitsui T et al (2008) Proliferation-promoting effect of platelet-rich plasma on human adipose-derived stem cells and human dermal fibroblasts. Plast Reconstr Surg 122:1352-1360

13. Lee SH, Jin SY, Sonq JS et al (2012) Paracrine effects of adipose-derived stem cells on keratinocytes and dermal fibroblasts. Ann Dermatol 24:136-143

14. Liang CC, Park AY, Guan JL (2007) In vitro scratch assay: a convenient and inexpensive method for analysis of cell migration in vitro. Nat Protoc 2:329-333

15. Lucarelli E, Beccheroni A, Donati D et al (2003) Platelet-derived growth factors enhance proliferation of human stromal stem cells. Biomaterials 24:3095-3100

16. Marques LF, Stessuk T, Camargo IC et al (2014) Platelet-rich plasma (PRP): methodological aspects and clinical applications. Platelets 26:101-113

17. Mills DC, Robb IA, Roberts GC (1968) The release of nucleotides, 5-hydroxytryptamine and enzymes from human blood platelets during aggregation. J Physiol 195:715-729

18. Park HB, Yang JH, Chung KH (2011) Characterization of the cytokine profile of platelet rich plasma (PRP) and PRP-induced cell proliferation and migration: $U p$ regulation of matrix metalloproteinase- 1 and -9 in $\mathrm{HaCaT}$ cells. Korean J Hematol 46:265-273

19. Pradhan L, Nabzdyk C, Andersen ND et al (2009) Inflammation and neuropeptides: the connection in diabetic wound healing. Expert Rev Mol Med 11:e2 
20. Shai A, Maibach HI (2005) Wound healing and ulcers of the skin. Springer, Berlin

21. Shan GQ, Zhang YN, Ma J et al (2013) Evaluation of the effects of homologous platelet gel on healing lower extremity wounds in patients with diabetes. Int $\mathbf{J}$ Low Extrem Wounds 12:22-29

22. Silva Meirelles L, Caplan AI, Nardi NB (2008) In search of the in vivo identity of mesenchymal stem cells. Stem Cells 26:2287-2299

23. Singer AJ, Clark RA (1999) Cutaneous wound healing. N Engl J Med 341:738-746

24. Walter MN, Wright KT, Fuller HR et al (2010) Mesenchymal stem cell-conditioned medium accelerates skin wound healing: an in vitro study of fibroblast and keratinocyte scratch assays. Exp Cell Res 316:1271-1281

25. Wong VW, Gurtner GC (2012) Tissue engineering for the management of chronic wounds: current concepts and future perspectives. Exp Dermatol 21:729-734
26. Xian LJ, Chowdhury SR, Saim AB et al (2015) Concentrationdependent effect of platelet-rich plasma on keratinocyte and fibroblast wound healing. Cytotherapy 17: 293-300

27. Xie X, Wang Y, Zhao C et al (2012) Comparative evaluation of MSCs from bone marrow and adipose tissue seeded in PRPderived scaffold for cartilage regeneration. Biomaterials 33:7008-7018

28. Yang HS, Shin J, Bhang SH et al (2011) Enhanced skin wound healing by a sustained release of growth factors contained in platelet-rich plasma. Exp Mol Med 43:622-629

29. Yoo KH, Jang IK, Lee MW et al (2009) Comparison of immunomodulatory properties of mesenchymal stem cells derived from adult human tissues. Cell Immunol 259:150-156

30. Zhao J, Hu L, Liu J et al (2013) The effects of cytokines in adipose stem cell-conditioned medium on the migration and proliferation of skin fibroblasts in vitro. Biomed Res Int 2013:578479 